\title{
A Long-term Forecast of Shallow Seismicity Based on the Global Strain Rate Map
}

\section{Peter Bird, ${ }^{1}$ Corné Kreemer, ${ }^{2}$ and William E. Holt ${ }^{3}$}

Online material: The gridded SHIFT/GSRM forecast for two representative threshold magnitudes, as well as files necessary to reproduce the computation or compute similar forecast maps for other threshold magnitudes.

\section{INTRODUCTION}

The Global Strain Rate Map (GSRM) of Kreemer et al. (2003) was the main result of Project II-8 of the International Lithosphere Program. The GSRM is a numerical velocity gradient tensor field model for the entire Earth's surface that describes the spatial variations of horizontal strain rate tensor components, rotation rates, and velocities. The model consists of 25 rigid spherical plates and $\sim 25,0000.6^{\circ}$ by $0.5^{\circ}$ deformable grid areas within the diffuse plate boundary zones (e.g., western North America, central Asia, Alpine-Himalaya belt). The model provides an estimate of the horizontal strain rates in diffuse plate boundary zones as well as the motions of the spherical caps. This is one of the first successful models of its kind that includes the kinematics of plate boundary zones in the description of global plate kinematics.

The vast majority of the data used to obtain the GSRM comes from horizontal velocity measurements obtained using Global Positioning System (GPS) measurements. The latest model version of May 2004 (i.e., GSRM version 1.2) includes 5,170 velocities for 4,214 sites worldwide (Holt et al. 2005). Most geodetic velocities are measured within plate boundary zones. The observed velocities are obtained from 86 different (mostly published) studies. The model includes additional constraints on the style (not magnitude) of the strain rate tensor inferred from moment tensors of shallow earthquakes. In addition, geologic strain rates in central Asia inferred from Quaternary faulting data are fit simultaneously with the geodetic velocities to improve the model there. See Kreemer et al. $(2000,2003)$ for more details.

1. Department of Earth and Space Sciences, University of California, Los Angeles

2. Nevada Bureau of Mines and Geology; Seismological Laboratory, University of Nevada, Reno

3. Department of Geosciences, State University of New York, Stony Brook
It was always a goal of the GSRM project to support longterm forecasts of seismicity based on tectonic deformation. Two recent developments make this especially timely. First, the Collaboratory for the Study of Earthquake Predictability (CSEP; Jordan et al. 2007) is accepting global models for prospective testing. To date, they have only registered global models that are based on smoothing of instrumental seismicity, so it would be valuable to compare results with a model based on tectonics. Second, the Global Earthquake Model project (http://www.globalquakemodel.org/) will soon create an update to the Global Seismic Hazard Map of Giardini et al. (1999), which was based primarily on instrumental and historical catalogs. The new map is likely to be based primarily on traces and slip rates of faults, so comparisons to seismicity models incorporating geodesy and plate tectonics should be illuminating and helpful.

To convert the GSRM to a forecast of long-term shallow seismicity, we apply the hypotheses, assumptions, and equations of Bird and Liu (2007), who referred to them as the "seismic hazard inferred from tectonics" (SHIFT) hypotheses: 1) The long-term seismic moment rate of any tectonic fault, or any large volume of permanently deforming lithosphere, is approximately that computed using the coupled seismogenic thickness (i.e., the seismic coupling coefficient times the seismogenic thickness) of the most comparable type of plate boundary; and 2) The longterm rate of earthquakes generated along any tectonic fault, or within any large volume of permanently deforming lithosphere, is approximately that computed from its moment rate (of the previous step) by using the frequency/magnitude distribution of the most comparable type of plate boundary.

In this conversion, we faced four conceptual and/or practical difficulties. First, the strain-rates available are not always the kinds of strain-rates that would be preferred. The strain-rates in GSRM were largely determined by GPS geodetic velocities, assumed plate and boundary geometry, and some local geologic and strain-direction constraints. Except where faults are creeping (or where they have many small earthquakes during the measurement period), the strain-rates inferred from differentiation of GPS velocities are dominantly elastic strain-rates associated with rising deviatoric stresses. However, the strainrates input to SHIFT calculations should ideally be long-term permanent strain-rates with no elastic components. Geodetic 


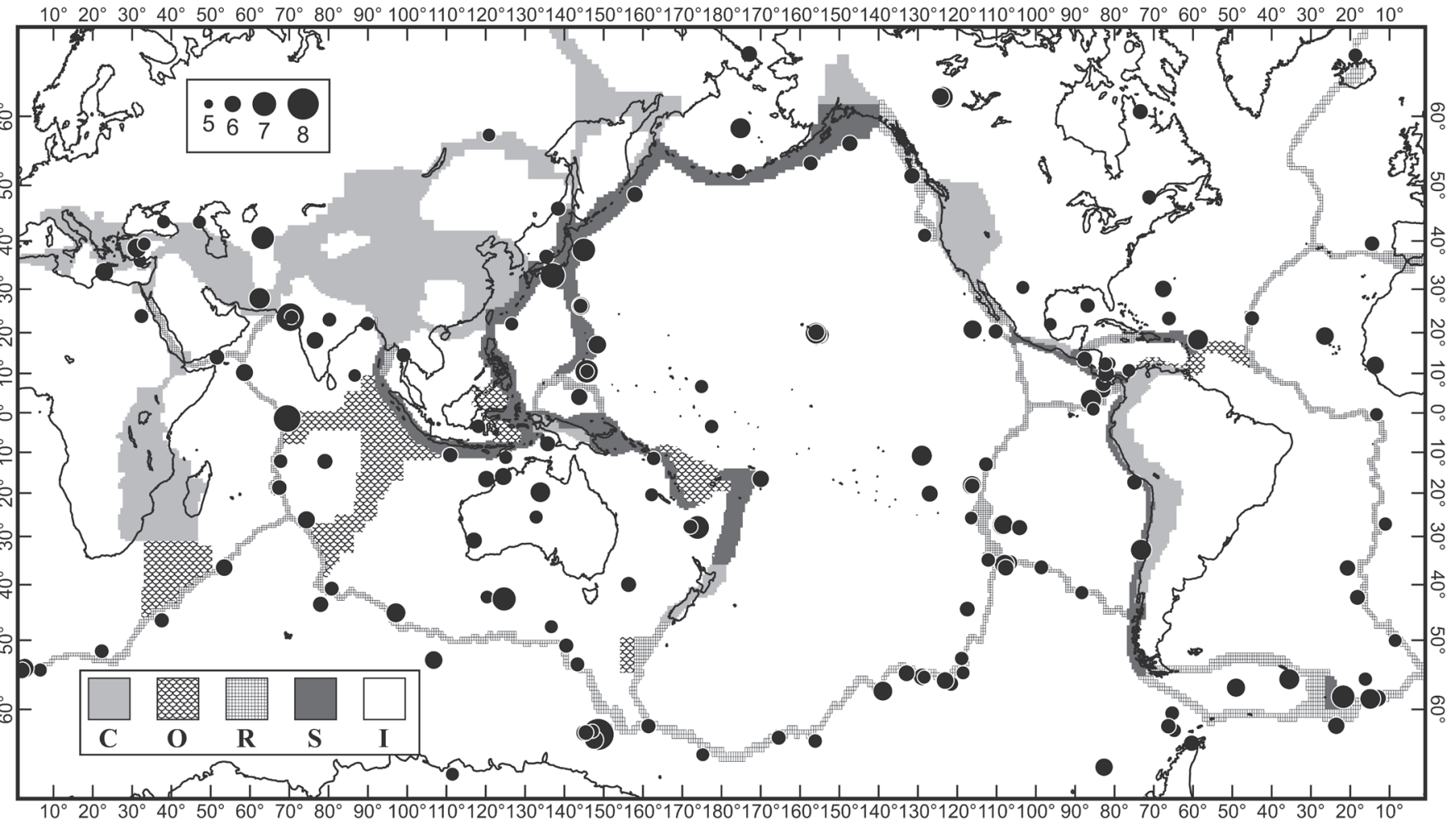

A Figure 1. Deformation regimes as defined by Kreemer et al. (2002): Subduction (S); diffuse Oceanic (0); Ridge-transform (R); Continental (C). Also shown are 189 shallow earthquakes above $m_{T}=5.66$ from the CMT catalog, 1977-2009.03, which did not fall into any of these regimes. These are considered intraplate (I) earthquakes.

strain-rates typically have smoother map patterns than longterm permanent strain-rates, which include singularities along fault traces. However, in a 2-D Earth-surface model based on plate-tectonic concepts, cross-boundary line integrals of these two kinds of strain-rate across a given plate boundary are the same, because both are equal to the relative plate velocity, regardless of timescale. The use of available GSRM strain-rates, which include some elastic components, should not greatly affect the total long-term seismicity computed in a SHIFT model, but only smooth its spatial distribution. On the scale of global maps and forecasts this smoothing is relatively insignificant compared to grid aliasing, digitization error, and other local error sources. Thus we disregard this distinction.

Second, GSRM treats plate interiors as perfectly rigid and predicts zero strain-rates in these regions. Yet a global seismicity forecast with zero rates in plate interiors would be both unrealistic and irresponsible. Our solution is to forecast a uniform low seismicity rate in all plate interiors, which is based on the collective frequency/magnitude distribution of these regions in the years covered by a reliable catalog. This makes our model formally a hybrid of two methods (SHIFT- and catalog-based), but as the two parts are spatially distinct there is little chance that these components will be confused.

Third, the basic SHIFT hypotheses do not specify how to decide which is the "most comparable type of plate boundary" for a given spatial grid point. This must be determined by subsidiary rules or hypotheses appropriate to the data and/or models available. We cannot use all of the decision rules suggested in Table 2 of Bird and Liu (2007) because they assumed that all subduction zones and spreading ridges were represented by discrete fault traces, which is not the case in GSRM. Fortunately, Kreemer et al. (2002) published a global map separating the deforming regions of GSRM into four deformation regimes: Subduction, Ridge-transform, diffuse Oceanic, and Continental (Figure 1). We use their map as the basis for assignments, and in some cases also use the tectonic style (e.g., normal-faulting, strike-slip, thrust-faulting, or mixed) of the local strain-rate tensor.

Finally, we found that our raw (uncorrected) forecast was seriously underpredicting global shallow seismicity (by a factor of 2) and that this was primarily due to underpredictions of subduction seismicity (by a factor greater than 3 ). We identified three quantifiable sources of underprediction in subduction zones: 1) inappropriate geometric factors in the momentrate formula for many thrust faults whose dips are much less than $45^{\circ} ; 2$ ) velocity-dependence of coupled seismogenic thickness in subduction zones inferred by Bird et al. (2009); and 3) time-dependence of global seismicity, which has increased since the calibration period of 1977-2002 studied by Bird and Kagan (2004). Compounding the corrections for these effects requires scaling-up the forecast seismicities of all grid points in the subduction-zone deformation regime by about a factor of 3. We apply smaller empirical correction factors to each of the other three deformation regimes. This yields an adjusted forecast that is reasonably consistent with the map-pattern and frequency/magnitude graph of the 33-year-old Global CentroidMoment-Tensor catalog. 
TABLE 1

Assignment of Most Comparable Type of Plate Boundary

\begin{tabular}{|lll|}
\hline $\begin{array}{l}\text { Deformation regime } \\
\text { (Kreemer } \text { et al. 2002) }\end{array}$ & $\begin{array}{l}\text { Plate boundary class } \\
\text { (Bird 2003) }\end{array}$ & Notes \\
\hline $\begin{array}{l}\text { Subduction (S) } \\
\text { Continental (C) }\end{array}$ & $\begin{array}{l}\text { Subduction zone (SUB) } \\
\text { Continental Transform Fault (CTF) or } \\
\text { Continental Rift Boundary (CRB) or } \\
\text { Continental Convergent Boundary (CRB) }\end{array}$ & $\begin{array}{l}\text { Assignment based on relative magnitude of } \dot{\varepsilon}_{\text {rr }} \\
\text { compared to horizontal principal strain rates, as } \\
\text { in Bird and Liu (2007) }\end{array}$ \\
Ridge-transform (R) & $\begin{array}{l}\text { Oceanic Transform Fault (OTF), plus either } \\
\text { Oceanic Spreading Ridge (OSR) or Oceanic }\end{array}$ & $\begin{array}{l}\text { Strain-rate tensor is partitioned into area-pre- } \\
\text { serving part (treated as OTF) and area-changing } \\
\text { part (treated as OSR or OCB) }\end{array}$ \\
& $\begin{array}{l}\text { Convergent Boundary (OCB) } \\
\text { Oceanic Convergent Boundary (OCB) }\end{array}$ & \\
diffuse Oceanic (0) & &
\end{tabular}

\section{DATA SETS}

The seismic catalog we refer to is the Centroid-Moment-Tensor (CMT) catalog (e.g., Ekström et al. 2005) formerly known as the Harvard CMT catalog but now known as the Global CMT catalog. It is available at http://www.globalcmt.org/ CMTsearch.html. We consider all shallow earthquakes, defined as those whose nominal centroid depth is $\leq 70 \mathrm{~km}$. We screen the catalog by a threshold seismic moment $\left(M_{T}=3.47 \times 10^{17} \mathrm{~N}\right.$ $\mathrm{m}$, equivalent to moment-magnitude threshold of $\left.m_{T}=5.66\right)$ to ensure that the subcatalog we use is nearly complete (Kagan 2003; Bird and Kagan 2004). We use the moment-to-magnitude conversion of Hanks and Kanamori (1979):

$$
m=\frac{2}{3}\left(\log _{10}(M)-9.05\right) .
$$

The four deformation regimes (Figure 1) defined by Kreemer et al. (2002) are defined in the electronic supplement as longitude/latitude rectangles of $0.6^{\circ} \times 0.5^{\circ}$ size, which collectively fill the domain of GSRM.

GSRM is represented by version 1.2 of May 2004, available at http://gsrm.unavco.org/. Specifically, we take horizontalplane (three-component) strain-rate tensors from the electronic supplement which gives average values, stated at the centers, of $0.6^{\circ} \times 0.5^{\circ}$ longitude/latitude rectangles forming a regular grid . Rectangles not described in this file are intraplate regions with zero assumed strain-rate.

Constants needed for the SHIFT calculations are obtained from Table 5 of Bird and Kagan (2004). This table has columns for each of seven classes of plate boundary, as defined in the PB2002 model of Bird (2003). It lists empirical estimates of: mean coupled thickness of seismogenic lithosphere $\langle c z\rangle$ (i.e., the mean value for each class of the seismic coupling coefficient times seismogenic thickness), elastic shear modulus $\mu$, corner magnitude $m_{\mathrm{c}}$ in the tapered Gutenberg-Richter frequency/ moment relation (Jackson and Kagan 1999; Kagan and Jackson 2000), asymptotic spectral slope $\beta$ in the same law, the number of shallow CMT earthquakes $N^{\mathrm{CMT}}$ (outside orogens and above threshold) associated with each class of plate bound- ary, and the threshold moment $M_{T}^{\mathrm{CMT}}$ used in counting those events.

\section{SHIFT CALCULATIONS}

To forecast the seismicity of one spatial grid point, we first characterize the strain-rate tensor. GSRM tensors are available on a regular grid in (longitude, latitude) $=(\phi, \theta)$ space representing the midpoints of the $0.6^{\circ} \times 0.5^{\circ}$ model cells, with the values at each grid point being the average for that cell. We sample the strain-rate tensor from the grid of GSRM values without any interpolation, merely selecting the value closest to each of our grid points. (This method should preserve both global and regional spatial averages of all strain-rate measures.)

Then we determine the vertical strain-rate $\dot{\varepsilon}_{r r}$ by invoking incompressibility:

$$
\dot{\varepsilon}_{\phi \phi}+\dot{\varepsilon}_{\theta \theta}+\dot{\varepsilon}_{r r}=0 .
$$

(This is one of the steps in which the strain-rate is assumed to be non-elastic.) Because $\dot{\varepsilon}_{r \phi}$ and $\dot{\varepsilon}_{r \theta}$ should vanish at a sheartraction-free surface, $\dot{\varepsilon}_{r r}$ is a principal strain-rate. The two principal strain-rates in the horizontal plane $\left(\dot{\varepsilon}_{1 \mathrm{~h}} \leq \dot{\varepsilon}_{2 \mathrm{~h}}\right)$ are:

$\dot{\varepsilon}_{1 \mathrm{~h}}=\frac{\dot{\varepsilon}_{\phi \phi}+\dot{\varepsilon}_{\theta \theta}}{2}-\sqrt{\dot{\varepsilon}_{\phi \theta}^{2}+\left(\dot{\varepsilon}_{\phi \phi}-\dot{\varepsilon}_{\theta \theta}\right)^{2} / 4} ;$
$\dot{\varepsilon}_{2 \mathrm{~h}}=\frac{\dot{\varepsilon}_{\phi \phi}+\dot{\varepsilon}_{\theta \theta}}{2}+\sqrt{\dot{\varepsilon}_{\phi \theta}^{2}+\left(\dot{\varepsilon}_{\phi \phi}-\dot{\varepsilon}_{\theta \theta}\right)^{2} / 4}$.

Lastly, these three principal strain-rates are alternately labeled as an ordered triplet:

$\dot{\varepsilon}_{1} \leq \dot{\varepsilon}_{2} \leq \dot{\varepsilon}_{3}$

Next we assign the "most comparable type of plate boundary" (from among the seven recognized in the PB2002 model of Bird 2003 and the analysis of Bird and Kagan 2004) by referring to the deformation regime map of Kreemer et al. (2002); in some 
cases we also make use of the tectonic character of the strainrate tensor. These assignments are summarized in Table 1.

If a point lies in the Subduction zone $(S)$ deformation regime, it is considered comparable to a Subduction (SUB) plate boundary and assigned parameters including coupled thickness $\langle c z\rangle=18 \mathrm{~km}$ and corner magnitude $m_{c}=9.58$.

If a point lies in the Continental (C) deformation regime, it is considered comparable to one of the three continental plate boundary types, based on the character of the strainrate tensor. Here we use the same rules as in Table 2 of Bird and Liu (2007): If $0 \leq \dot{\varepsilon}_{r r} \leq 0.364 \dot{\varepsilon}_{2 \mathrm{~h}}$ or $0.364 \dot{\varepsilon}_{1 \mathrm{~h}} \leq \dot{\varepsilon}_{r r}<0$ it is considered comparable to a Continental Transform Fault (CTF) plate boundary and assigned parameters including $\langle c z\rangle=8.6 \mathrm{~km}$ and $m_{\mathrm{c}}=8.01$. If $\dot{\varepsilon}_{r r}$ is more positive, indicating a dominance of thrust faulting, it is considered comparable to a Continental Convergent Boundary (CCB) plate boundary and assigned parameters including $\langle c z\rangle=18 \mathrm{~km}$ and $m_{\mathrm{c}}=8.46$. If $\dot{\varepsilon}_{r r}$ is more negative, indicating a dominance of normal faulting, it is considered comparable to a Continental Rift Boundary (CRB) plate boundary and assigned parameters including $\langle c z\rangle=3 \mathrm{~km}$ and $m_{\mathrm{c}}=7.64$.

If a point lies in a Ridge-transform ( $R$ ) deformation regime, we distinguish four cases using the strain-rate tensor:

1. $\dot{\varepsilon}_{1 \mathrm{~h}} \geq 0$ is a case of simple spreading with no transform faulting; it is considered comparable to an Oceanic Spreading Ridge (OSR) plate boundary and assigned parameters including $\langle c z\rangle=0.13 \mathrm{~km}$ and $m_{\mathrm{c}}=5.86$.

2. $\left(\dot{\varepsilon}_{1 \mathrm{~h}} \times \dot{\varepsilon}_{2 \mathrm{~h}}\right)<0$ with $\left(\dot{\varepsilon}_{1 \mathrm{~h}}+\dot{\varepsilon}_{2 \mathrm{~h}}\right) \geq 0$ is a case of spreading plus transform faulting; we partition the strain-rate into a portion $\left(\dot{\varepsilon}_{1 \mathrm{~h}}^{\text {ridge }}=0, \dot{\varepsilon}_{2 \mathrm{~h}}^{\text {ridge }}=\dot{\varepsilon}_{1 \mathrm{~h}}+\dot{\varepsilon}_{2 \mathrm{~h}}\right)$ that is comparable to OSR as above, plus a portion $\left(\dot{\varepsilon}_{1 \mathrm{~h}}^{\text {transform }}=\dot{\varepsilon}_{1 \mathrm{~h}}, \dot{\varepsilon}_{2 \mathrm{~h}}^{\text {transform }}=-\dot{\varepsilon}_{1 \mathrm{~h}}\right)$ that is comparable to an Oceanic Transform Fault (OTF) plate boundary and is assigned parameters including $\langle c z\rangle=1.8 \mathrm{~km}$ and $m_{\mathrm{c}}=6.55$.

3. $\left(\dot{\varepsilon}_{1 \mathrm{~h}} \times \dot{\varepsilon}_{2 \mathrm{~h}}\right)<0$ with $\left(\dot{\varepsilon}_{1 \mathrm{~h}}+\dot{\varepsilon}_{2 \mathrm{~h}}\right)<0$ is a case of thrustfaulting plus transform faulting; we partition the strainrate into a portion $\left(\dot{\varepsilon}_{1 \mathrm{~h}}^{\text {thrust }}=\dot{\varepsilon}_{1 \mathrm{~h}}+\dot{\varepsilon}_{2 \mathrm{~h}}, \dot{\varepsilon}_{2 \mathrm{~h}}^{\text {thrust }}=0\right)$ that is considered comparable to an Oceanic Convergent Boundary (OCB) with $\langle c z\rangle=3.8 \mathrm{~km}$ and $m_{c}=8.04$, plus a portion $\left(\dot{\varepsilon}_{1 \mathrm{~h}}^{\text {transform }}=-\dot{\varepsilon}_{2 \mathrm{~h}}, \dot{\varepsilon}_{2 \mathrm{~h}}^{\text {transform }}=\dot{\varepsilon}_{2 \mathrm{~h}}\right)$ that is comparable to OTF as above.

4. $\dot{\varepsilon}_{2 h}<0$ is a case of thrust faulting with no transform faulting; it is considered comparable to an OCB as above. If a point lies in a diffuse Oceanic $(\mathrm{O})$ deformation regime, it is considered comparable to an Oceanic Convergent Boundary (OCB) plate boundary and assigned parameters including coupled thickness $\langle c z\rangle=3.8 \mathrm{~km}$ and corner magnitude $m_{\mathrm{c}}=8.04$. (Note that these points are generally far from spreading ridges where the oceanic lithosphere is thicker, so we think the larger coupled thickness and higher corner magnitude of OCB may be appropriate even if the deformation is not compressional.)

If a point does not lie in any of the four deformation regimes, it is considered an Intraplate point. Intraplate seismicity is forecast differently, as discussed in the next section.
Once the seismicity parameters such as coupled thickness $\langle c z\rangle$ and elastic shear modulus $\mu$ have been assigned, we compute the expected long-term seismic moment rate per unit area $A$ using Equation 7B of Bird and Liu (2007):

$$
\frac{\dot{M}}{A}=\langle c z\rangle_{\mu}\left\{\begin{array}{cc}
2 \dot{\varepsilon}_{3} ; & \text { if } \dot{\varepsilon}_{2}<0, \text { or } \\
-2 \dot{\varepsilon}_{1} ; & \text { if } \dot{\varepsilon}_{2} \geq 0
\end{array}\right. \text {. }
$$

This equation is based on a kinematic model: that volumepreserving deformation is equivalent to slip on many minor virtual faults falling into (up to) two conjugate sets. The more active conjugate fault set bisects the angles between the principal strain-rate axes $\pm \hat{\varepsilon}_{1}$ and $\pm \hat{\varepsilon}_{3}$; the less active conjugate fault set bisects the angles between principal strain-rate axes $\pm \hat{\varepsilon}_{2}$ and $\pm \hat{\varepsilon}_{3}$ (if $\dot{\varepsilon}_{2}<0$ ) or between $\pm \hat{\varepsilon}_{1}$ and $\pm \hat{\varepsilon}_{2}$ (if $\dot{\varepsilon}_{2} \geq 0$ ). The factor of \pm 2 appearing in Equation 5 is the smallest coefficient possible and comes from the assumption that the virtual fault planes make angles of $45^{\circ}$ with the principal strain-rate axes; this point will be discussed further in a later section. Naturally, this formula also assumes that the strain-rates are long-term permanent strain rates, and not elastic.

Note that published formulas for converting strain-rate to seismic moment rate have not always agreed. The prediction of moment rate in Equation 5 is identical to that of Savage and Simpson (1997). Another common formula (Table 5 of Pancha et al. 2006) has been the one used by Anderson (1979, if efficiency factor $k=1)$, Ward $(1994,1998 \mathrm{a}, \mathrm{b})$, and Kreemer et al. (2002); this will be equal in typical cases where the principal strain-rate with greatest magnitude is horizontal; however, our Equation 5 gives a slightly larger minimum moment rate in those relatively rare cases where the principal strain-rate with greatest magnitude is vertical.

Once the long-term seismic moment rate $(\dot{M})$ of a grid point is determined, its expected long-term shallow seismicity rate is obtained in two steps. First, we divide the long-term moment rate by the model moment rate (integral of best-fitting tapered Gutenberg-Richter distribution) of the appropriate Bird and Kagan (2004) subcatalog of the CMT catalog and multiply by the number of events in that subcatalog to determine the rate of earthquakes at the grid point that will exceed the threshold magnitude of that subcatalog:

$$
\dot{N}\left(m>m_{\mathrm{T}}^{\mathrm{CMT}}\right)=\left(\dot{M} / \dot{M}^{\mathrm{CMT}}\right) \dot{N}^{\mathrm{CMT}} .
$$

Then we adjust the forecast rate to any desired threshold magnitude $m_{\mathrm{T}}$ by using the tapered Gutenberg-Richter model (see also Equation 9 of Bird and Kagan 2004):

$$
\begin{aligned}
\dot{N}\left(m>m_{\mathrm{T}}\right)= & \dot{N}\left(m>m_{\mathrm{T}}^{\mathrm{CMT}}\right)\left(\frac{M\left(m_{\mathrm{T}}\right)}{M\left(m_{\mathrm{T}}^{\mathrm{CMT}}\right)}\right)^{-\beta} \\
& \times \exp \left(\frac{M\left(m_{\mathrm{T}}^{\mathrm{CMT}}\right)-M\left(m_{\mathrm{T}}\right)}{M\left(m_{\mathrm{c}}\right)}\right)
\end{aligned}
$$




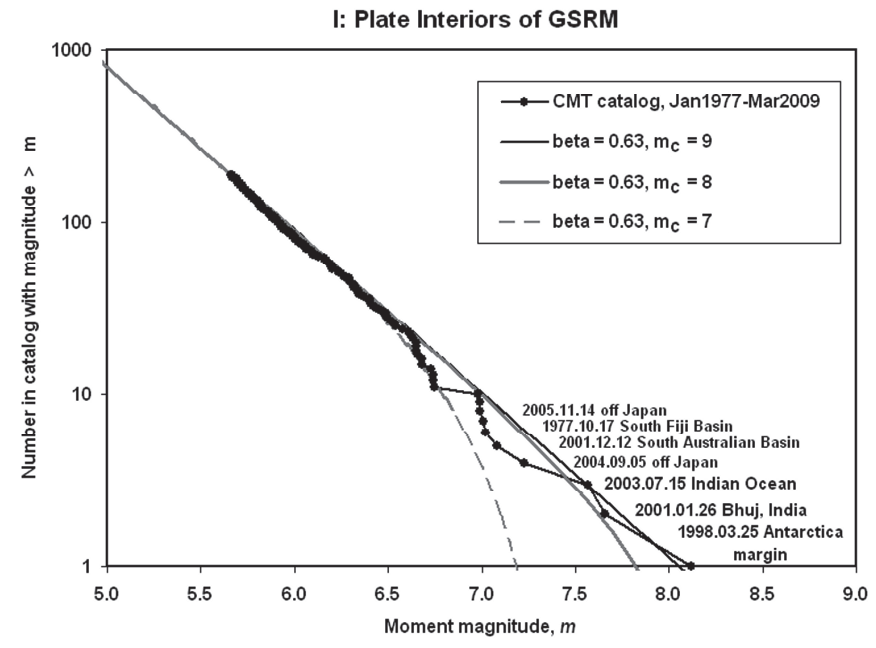

A Figure 2. Frequency/magnitude plot of the 189 shallow intraplate earthquakes from Figure 1. Also shown are three tapered Gutenberg-Richter model curves (Jackson and Kagan 1999; Kagan and Jackson 2000). All models have the same asymptotic spectral slope of $\beta=0.63$, but differ in the choice of corner magnitude $m_{c}$. While the match to the curve with corner magnitude of 9 appears best, it must be noted that this depends on the size of the single largest earthquake.

where $m_{c}$ is the corner magnitude for seismicity of the analog plate boundary type.

These calculations are implemented in program SHIFT_ GSRM.f90, whose Fortran 90 source code is inlcuded in the electronic supplement.

\section{INTRAPLATE SEISMICITY}

Because GSRM does not estimate tectonic strain-rates of plate interiors, we use a simple empirical-averaging method. Figure 1 shows the epicentroids of 189 CMT earthquakes $(z \leq 70 \mathrm{~km}$, $m \geq 5.66,1977.01 .01-2009.03 .31)$ that fell within the intraplate regions of the GSRM model. They represent only $2.7 \%$ of shallow seismicity. As the intraplate area is $4.35 \times 10^{14} \mathrm{~m}^{2}$ and the catalog duration was $1.02 \times 10^{9} \mathrm{~s}$, the mean intraplate seismicity at this threshold is $4.27 \times 10^{-22} \mathrm{~m}^{-2} \mathrm{~s}^{-1}$. We use this as the forecast seismicity at all intraplate points when the desired threshold is $m_{\mathrm{T}}=5.66$.

Extrapolation of intraplate seismicity to other thresholds requires estimating the corner-magnitude $\left(m_{c}\right)$ and spectral slope ( $\beta$ ) parameters of the tapered Gutenberg-Richter frequency/magnitude distribution. Figure 2 shows one possible fit, with $\beta=0.63$ and $m_{c}=9$, which we adopted for purposes of this forecast. (Note that the corner magnitude is not well constrained, except that it must be greater than 8.) With these parameters, the projection is that there should be, on average, one intraplate earthquake of $m>8$ every 29 years. The CMT catalog includes just one: 1998.03.25 $m 8.12$ near the Balleny Islands off the margin of Antarctica.

\section{RETROSPECTIVE COMPARISON AND ADJUSTMENT}

The forecasting methods described above are relatively simple and objective, and up to this point they have involved no adjustable parameters. They result in a raw global long-term forecast of shallow seismicity (above $m_{\mathrm{T}}=5.66$, for any window of 32.25-year length) which integrates around the Earth to a total of 3,201 earthquakes. However, the shallow earthquake count from the CMT catalog in 1977.01.01-2009.03.31 was 6,983 earthquakes. Clearly some part(s) of our algorithm is (are) badly underpredicting seismicity. The discrepancy is coming from the ocean floors, as detailed in the breakdown by deformation regime shown in Table 2.

The close agreement of forecast and actual seismicity in continental areas probably reflects the good geodetic control of velocities and strain-rates on land. Because model GSRM (strain-rates) and model PB2002 (plate velocities) were both influenced by the same geodetic studies, they tend to have very similar overall rates of continental deformation. The PB2002 model was used by Bird and Kagan (2004) to derive seismicity parameters of continental (CCB, CTF, and CRB) plate boundaries from the CMT catalog. These seismicity parameters were then used in this study to forecast seismicity rates on continents, which were then checked against CMT. Therefore, the agreement seen here is primarily a positive consistency check. However, it is slightly more significant than that, because Bird and Kagan (2004) excluded complex continental "orogens" from their calibration regions, but in GSRM and in the present comparison they are included.

The generally poorer agreement in the three oceanic deformation regimes $(\mathrm{O}, \mathrm{R}, \mathrm{S})$ may reflect poorer geodetic control (at least for $\mathrm{O}$ where it is limited to selected islands), leading to more widespread differences between kinematic models GSRM and PB2002. Where these models have very different overall rates of deformation, our consistency checks might be expected to fail. Another complication in the seafloor domain is that there are some indications that seismic coupling may depend on fault slip rates: on spreading ridges (Kreemer et al. 2002; Bird et al. 2002; Bird et al. 2009), on oceanic transform faults (Bird et al. 2002; Bird and Kagan 2004), and in subduction zones (Bird et al. 2009). Because the GSRM kinematic model does not have discrete faults with modeled slip rates, it is not possible to use any of these velocity-dependent models in the present seismicity forecast. (Instead, velocity-independent mean seismicity parameters from Bird and Kagan 2004 are applied universally to seafloor strain-rates within each regime.) Another possible complication relates to the distinction between longterm strain-rates (preferred for SHIFT) and partially elastic strain-rates (provided by GSRM), and the way in which this leads to spatial smoothing. Several areas of the deformationregime map of Kreemer et al. (2002) have thin belts of S regime paralleled by belts of $\mathrm{C}$ regime: Andes, Kamchatka, Ryukyu Islands, and central Alaska. If the elastic-smoothing effect has displaced some high strain-rates across these boundaries from $\mathrm{S}$ to $\mathrm{C}$, then this would help to explain the deficient forecast in 


\begin{tabular}{|lccc|}
\hline \multicolumn{4}{c|}{ TABLE 2 } \\
& Empirical Corrections Incorporated in this Forecast $\left(\boldsymbol{m}_{\boldsymbol{T}}=\mathbf{5 . 6 6 )}\right.$ \\
\hline $\begin{array}{l}\text { Deformation regime } \\
\text { (Kreemer } \text { et al. 2002): }\end{array}$ & $\begin{array}{c}\text { uncorrected forecast } \\
\text { for 32.25 years: }\end{array}$ & $\begin{array}{c}\text { actual earthquakes, } \\
\text { 1977-2009.03: }\end{array}$ & Correction factor: \\
\hline Subduction (S) & 1,310 & 4,498 & 3.434 \\
diffuse Oceanic (O) & 119 & 238 & 2.000 \\
Ridge-transform (R) & 764 & 1,237 & 1.619 \\
Continental (C) & 820 & 821 & 1.001 \\
\hline
\end{tabular}

domain S. (On the other hand, it would suggest that the agreement found for regime $\mathrm{C}$ might be only a coincidence in which two errors offset each other.) None of these issues will be easy to resolve.

First we will focus on the great discrepancy in seismicity of subduction zones and examine three partial explanations which can each be roughly quantified. We will show that these three systematic errors have interacted to reduce our SHIFT forecast of subduction seismicity by about a factor of 3 . This provides justification for applying an empirical correction factor of similar size to the subduction-zone deformation regime portion of this forecast.

\section{Effect of Fault Dip}

Consider a straight thrust fault trace or subduction zone parallel to axis $y$ on a flat Cartesian Earth. Let relative plate velocity be orthogonal at rate $\Delta v_{x}$. If the fault dip is $\alpha$ and the thickness of seismogenic lithosphere is $z$, then potentially seismogenic fault area per unit length of trace or trench is $z / \sin \alpha$. The fault slip rate is $\Delta v_{x} / \cos \alpha$. (This is because convergence generates relative vertical velocity of $\Delta v_{z}=\Delta v_{x} \tan \alpha$ at the fault, and by assumption $\Delta v_{y}=0$ in this case, so $\left.\sqrt{\Delta v_{x}^{2}+\Delta v_{y}^{2}+\Delta v_{z}^{2}}=\Delta v_{x} / \cos \alpha\right)$. In this model, the seismic moment rate per unit length of trace or trench is $\Delta v_{x}\langle c z\rangle \mu /(\sin \alpha \cos \alpha)$. But, if we distribute the relative velocity across a plate boundary zone of width $w$, as $\dot{\varepsilon}_{1 \mathrm{~h}}=\dot{\varepsilon}_{x x}=\Delta v_{x} / w$, then SHIFT continuum Equation (5) predicts a seismic moment rate of $2 \Delta v_{x}\langle c z\rangle \mu$ per unit length of plate boundary. These rates are only equivalent for $\alpha=45^{\circ}$. For a typical dip of $\alpha=14^{\circ}$ in the seismogenic part of the interplate shear zone in a subduction zone (Bird and Kagan 2004), the SHIFT continuum formula underpredicts by a large factor of 2.13. For a dip of $\alpha=20^{\circ}$ that might describe well-established thrusts in continental convergent settings, the SHIFT continuum formula underpredicts by a factor of 1.56 . (For normal faulting, the effect is less important: the correction factor for $\alpha=55^{\circ}$ is only 1.06 .)

\section{Possible Velocity-dependence of Subduction Seismicity}

Because GSRM does not have discrete subduction zone faults with defined relative velocities, we have used the SHIFT continuum Equation (5) with the mean seismicity parameters of Bird and Kagan (2004) for all of deformation regime S. This is consistent with the finding of a linear relationship between velocity and seismicity by Kreemer et al. (2002). However, Bird et al. (2009) re-examined the relationship between relative plate velocity and shallow seismicity of subduction zones (using a different list of subduction zones and a different kinematic model) and found some velocity-dependence. Specifically, the latter study concludes that earthquake rates (per unit of trench length, per unit of relative plate velocity) increase by a factor of about 2 at a critical plate velocity of approximately 66 $\mathrm{mm} / \mathrm{a}$. Bird et al. (2009) suggested that mean coupled thickness $\langle c z\rangle=18 \mathrm{~km}$ for all subduction zones conceals a contrast between $c z$ of about $10.6 \mathrm{~km}$ for slower subduction zones and $c z$ of about $21.7 \mathrm{~km}$ for faster subduction zones.

The relevance of this distinction is that most of the subduction zones included in deformation regime $S$ are faster subduction zones according to this criterion. Many of the slower convergent plate boundaries that Bird et al. (2009) considered to be slow subduction zones were included as parts of continental regime $\mathrm{C}$ or diffuse oceanic regime $\mathrm{O}$ by Kreemer $e t a l$. (2002). This is probably the most important explanation for the difference in the findings of these two studies. Regardless of which term is most appropriate to describe the slower convergent plate boundaries, it now seems that many of the faster subduction zones in tectonic regime $S$ should be described by the higher coupled thickness of $21.7 \mathrm{~km}$ rather than the mean value of $18 \mathrm{~km}$. This suggests a correction factor of as much as $21.7 / 18=1.21$, which should be compounded with the dipdependent correction discussed above.

Bird et al. (2009) also inferred an increase in coupled thickness and seismicity of continental convergent boundaries at a different critical velocity. However, deformation regime $\mathrm{C}$ of Kreemer et al. (2002) includes both slower and faster continental convergent boundaries; therefore, there is probably no need for a correction. (Again, fault-specific corrections cannot be attempted where discrete faults and their slip rates were not modeled.)

\section{Time-dependence of Global Seismicity}

Bird and Kagan (2004) performed their calibration study using years 1977.01.01-2002.09.30, during which the mean rate of shallow earthquakes above $m_{\mathrm{T}}=5.66$ was 206 earthquakes/ year. However, over the whole catalog period of 1977.01.012009.03.31 now available, the rate is larger, at 217 earthquakes/ year. In the last six years, there have been about 340 more shallow earthquakes than we expected based on extrapolation of 1977-2002 rates. More than half of this increase is due to the approximately 165 CMT aftershocks of the 2004.12.26 m 9.03 Banda Aceh earthquake (including secondary aftershocks of two $m 8$ primary aftershocks), with small contributions from the seven CMT aftershocks of the 2008.05.12 
m 7.94 Wenchuan earthquake, the six CMT aftershocks of the 2002.11.03 $m$ 7.88 Denali earthquake, and the six CMT aftershocks of the 2005.10.08 $\mathrm{m} 7.61 \mathrm{Kashmir}$ earthquake. (This list is limited to earthquakes that significantly increased the apparent long-term moment rates for their respective plate boundary classes.) The other half of the seismicity increase is harder to locate, but it appears that there has been an increase in rates in other subduction zones as well.

Considering that overall shallow earthquake rates are now known to be $5.1 \%$ larger than in the period studied by Bird and Kagan (2004), with most of the increase occurring in subduction zones, and that subduction zones contribute about $63 \%$ of global seismicity (Kreemer et al. 2002), it appears that Bird and Kagan (2004) may have underestimated the coupled thicknesses and seismicities of these plate boundaries by an additional factor of about $5.1 \% / 63 \%=8.1 \%$. This factor should be compounded with the two others discussed above.

Considering all the systematic contributions above, it appears that our initial uncorrected seismicity forecast for subduction zones was artificially depressed by a factor of about $2.13 \times 1.21 \times 1.081=2.79$. In the adjusted version of our forecast, we compensate by introducing a "subduction factor" that amplifies the seismicity of all subduction zones uniformly (relative to the SHIFT formulas detailed in a previous section). By setting this subduction factor to empirical value $4498 / 1310=3.434$ (which is $23 \%$ higher than the estimated 2.79), we produce a forecast for the subduction-zone deformation regime that matches the corresponding shallow seismicity recorded by CMT in 1977-2009.03 (Table 2). One possible reason why this extra increment (i.e., 3.434/2.79 $\cong 1.23$ ) is needed could be that the PB2002 kinematic model (used by Bird and Kagan 2004 to calibrate seismicity factors) has greater amounts of back-arc spreading and thus systematically higher rates of subduction than the GSRM kinematic model. Unfortunately, such comparisons cannot help us to decide which kinematic model is more accurate.

In the diffuse Oceanic $(\mathrm{O})$ deformation regime, we require an empirical correction factor with size 238/119 $=2$. The need for this factor-of-two correction probably indicates a greater mean coupled seismogenic thickness (e.g., $7.6 \mathrm{~km}$ vs. 3.8 $\mathrm{km}$ ) in the diffuse Oceanic deformation regime of Kreemer et al. (2002) than in the Oceanic Convergent Boundary (OCB) plate boundary class of Bird (2003) and Bird and Kagan (2004), which we used here as the "most comparable type of plate boundary." In turn, this probably arises from geographic discrepancies in their respective definitions. The diffuse Oceanic deformation regime (Figure 1) is dominated by three regions in which relatively old and stiff oceanic lithosphere is being deformed to create new plate boundaries: in the east-central Indian Ocean, in the west-central Atlantic, and southeast of South Africa. In contrast, the OCB plate boundary steps used by Bird and Kagan (2004) in their seismicity calibration excluded the first two of these regions (because they had been labeled as complex "orogens"), but also differed by including a widely distributed set of seafloor faults along ordinary spreading ridges with transpressional relative velocities (estimated to differ by more than $20^{\circ}$ from fault trend). (Others might label these faults as "transform faults" based on either a different kinematic model or a different kinematic criterion.) It is likely that the mean lithospheric age is systematically younger for this group of transpressive faults.

In the Ridge-transform (R) deformation regime, we require a slightly smaller empirical correction factor of $1,237 / 764=1.619$. This is harder to explain, because the geographic domain of the $\mathrm{R}$ deformation regime (Figure 1) closely approximates the union of the Oceanic Spreading Ridge (OSR) and Oceanic Transform Fault (OTF) plate boundary classes used by Bird and Kagan (2004) in their seismic calibration. One difference is that the geographic footprint of the union of the OSR and OTF regimes (OSR VOTF) includes some backarc spreading ridges (e.g., in the Tonga-Kermadec, Mariana, and Ryukyu systems) which were not recognized as parts of regime R. However, we have no evidence that back-arc spreading ridges are different from other spreading ridges in terms of their seismicity parameters. Another consideration could be that the $\mathrm{R}$ deformation regime includes transpressive seafloor faults which Bird (2003) placed in boundary class OCB rather than OTF. However, in our algorithm for this forecast we used OCB seismicity parameters for any transpressive elements of strain-rate tensors of grid points in the R regime, so we have attempted to correct for this effect.

The correction factor applied in the Continental (C) deformation regime is $821 / 820=1.001$, which is not significantly different from unity. This factor is included only for formal consistency of method across all four deformation regimes.

\section{SHIFT/GSRM FORECAST}

Clearly this forecast has some problems and deficiencies. Still, it should be of interest because it is the first global forecast whose map pattern has a long-term tectonic basis. This forecast may be conveniently called the "SHIFT/GSRM" long-term forecast of shallow seismicity, because it layers the SHIFT assumptions and equations onto the kinematic foundation of GSRM. (This naming convention allows for alternative forecasts to be constructed on the kinematic basis of GSRM in the future.)

Specifically, it is a set of global maps represented by gridded values $\left(89.95^{\circ} \mathrm{S}\right.$ to $89.95^{\circ} \mathrm{N}, 0.05^{\circ} \mathrm{E}$ to $360.05^{\circ} \mathrm{E}$, in $0.1^{\circ}$ steps) of the forecast rate of shallow earthquake epicenters per square meter per second (including aftershocks) above threshold. Each map (for a particular threshold) corresponds to a separate file in “.grd" format (defined at http://peterbird.name/guide/grd_format.htm). Two representative thresholds $\left(m_{\mathrm{T}}=5.66,8.00\right)$ are represented by Figures 3 and 4 and also shown in the electronic supplement. It is easy to compute gridded seismicity values for other thresholds with the program described above.

This forecast is "long-term" in the sense that it does not include time-dependent effects such as aftershock swarms or interevent triggering (either static or dynamic). In principle, the forecast seismicity fields are stationary up to very long timescales of a million years or more, in which plate geometries change. In practice, the forecast is likely to be updated in response to new 


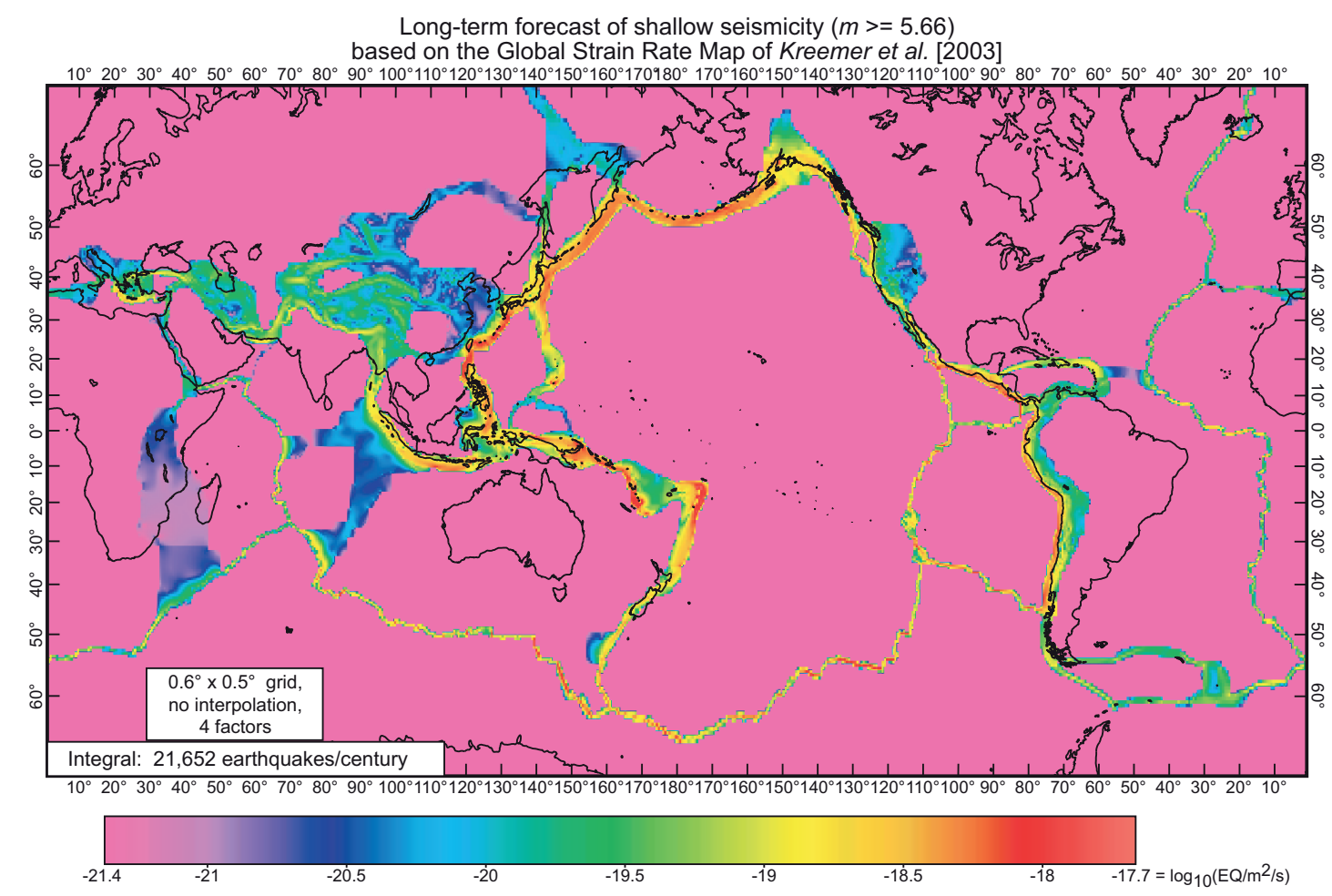

$\Delta$ Figure 3. SHIFT/GSRM global long-term forecast of the rates of shallow earthquakes above $m_{T}=5.66$. This model has been adjusted to match global shallow earthquake rates from CMT in 1977-2009.03 by using one free parameter for each of four deformation regimes, and one for the intraplate area. Rates are expressed as earthquakes per square meter per second, including aftershocks. Coloring of the map employs a logarithmic scale to express variations across almost four orders of magnitude from peak subduction-zone rates to intraplate rates. Mercator projection.

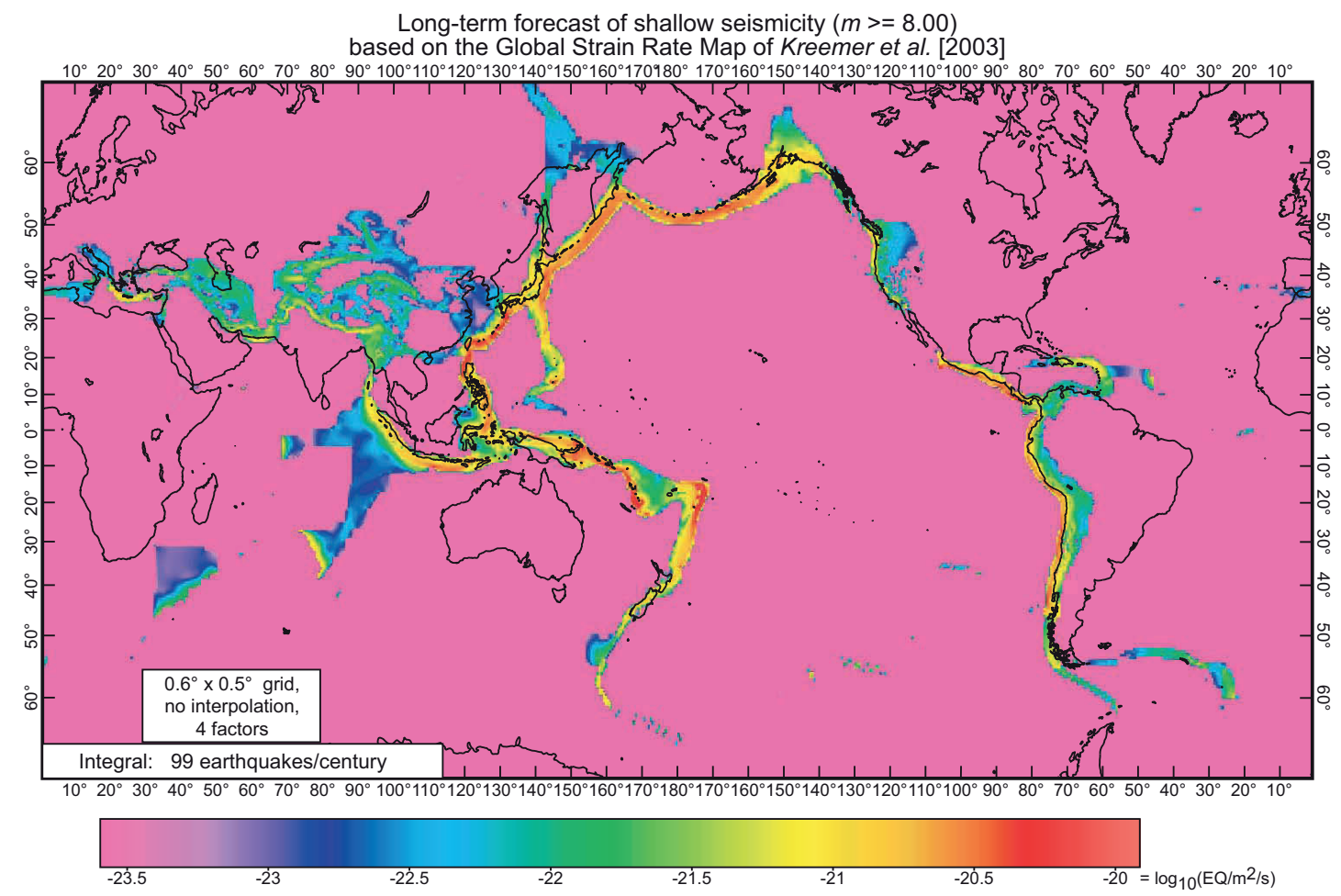

$\Delta$ Figure 4. SHIFT/GSRM global long-term forecast of the rates of shallow earthquakes above $m_{T}=8.00$. Conventions as in Figure 3. In this model, mid-ocean spreading ridges and ideal oceanic transforms do not contribute to seismicity at magnitudes above 8 , while the contributions of continental rifts and transpressive oceanic transforms are generally small. 
geodetic and geologic studies, small changes in the calibration constants as the available catalog gets longer, and/or new ideas about assumptions and numerical methods. Therefore, it could also be called an "indefinite-term" or "open-window" forecast. This means that it is meaningful to compare it to seismic catalogs retrospectively as well as prospectively, while stipulating that prospective testing will ultimately be decisive because retrospective comparisons involve elements of circularity.

Figure 5 shows the frequency/magnitude curve for the forecast, in retrospective comparison to CMT data. As described above, our forecast has been normalized to the CMT rate for $m_{\mathrm{T}}=5.66$. The asymptotic spectral slope of both curves is about $\beta \cong 0.63$. Both have a downward curvature, because regions with modest corner magnitudes like spreading ridges and oceanic transforms do not contribute any high-magnitude seismicity (Figure 4). Data and forecast agree well up to magnitude 7.7. However, the forecast curve is systematically above the CMT curve for magnitudes above 7.7, with the mismatch increasing to a factor-of-2 at magnitude 9. This suggests that some of the corner magnitudes (from Bird and Kagan 2004) used in our computation may be too high. On the other hand, these corner magnitudes were based on a catalog by Pacheco and Sykes (1992) extending back to the beginnings of instrumental catalogs in 1900, so it may be the 32-year interval of the CMT data that is anomalous relative to the 77-year interval that preceded it. Resolving this question statistically will be difficult because it is not clear what distribution describes earthquake counts sampled from a tectonically stationary process. Sample errors bars in Figure 5 show that a match of high-magnitude rates would be rejected with 95\%-confidence if earthquakes were independent and earthquake counts followed the binomial dis- tribution; however, Kagan (forthcoming) has shown that real earthquake-count distributions are broader.

In another retrospective test of this forecast, we compute cumulative spatial distribution functions for both forecast and actual seismicity (Figure 6) and the cross-plot of cumulative actual seismicity against cumulative forecast seismicity (Figure 7). To prepare these, we sort the $0.1^{\circ} \times 0.1^{\circ}$ grid cells of one forecast map $\left(m_{\mathrm{T}}=5.66\right)$ in order of increasing forecast earthquake rate. (At the same time, we sort the corresponding numbers of actual CMT earthquakes in each cell-passively, and without using these integers for ordering.) Then we integrate each list to produce monotonically increasing lists of cumulative area, cumulative forecast seismicity, and cumulative actual seismicity. The two spatial distribution functions (SDFs) are defined as cumulative number of forecast (or actual) earthquakes per 32.25 years, as a function of cumulative area. Clearly, these two SDFs are similar (Figure 6). Plotting one against the other (Figure 7) produces a curve that should ideally be linear, and is actually quite close. The discrepancy seen at the lower left of Figure 7 (i.e., where cumulative forecast earthquakes $=565$, but actual cumulative earthquakes $=1,005)$ indicates that some of the forecast low-seismicity areas (blue areas in Figure 3) actually have relatively concentrated earthquakes: central East Africa rift, east-central Indian Ocean, and Italian peninsula. The causes may include both the elastic smoothing effect in GSRM that was previously discussed, and local shortages of geodetic control that would be needed to define concentrations in strain-rate.

This forecast will be submitted to CSEP for a new round of prospective testing of global forecasts scheduled to begin in 2010. If it is relatively successful this forecast could be con-

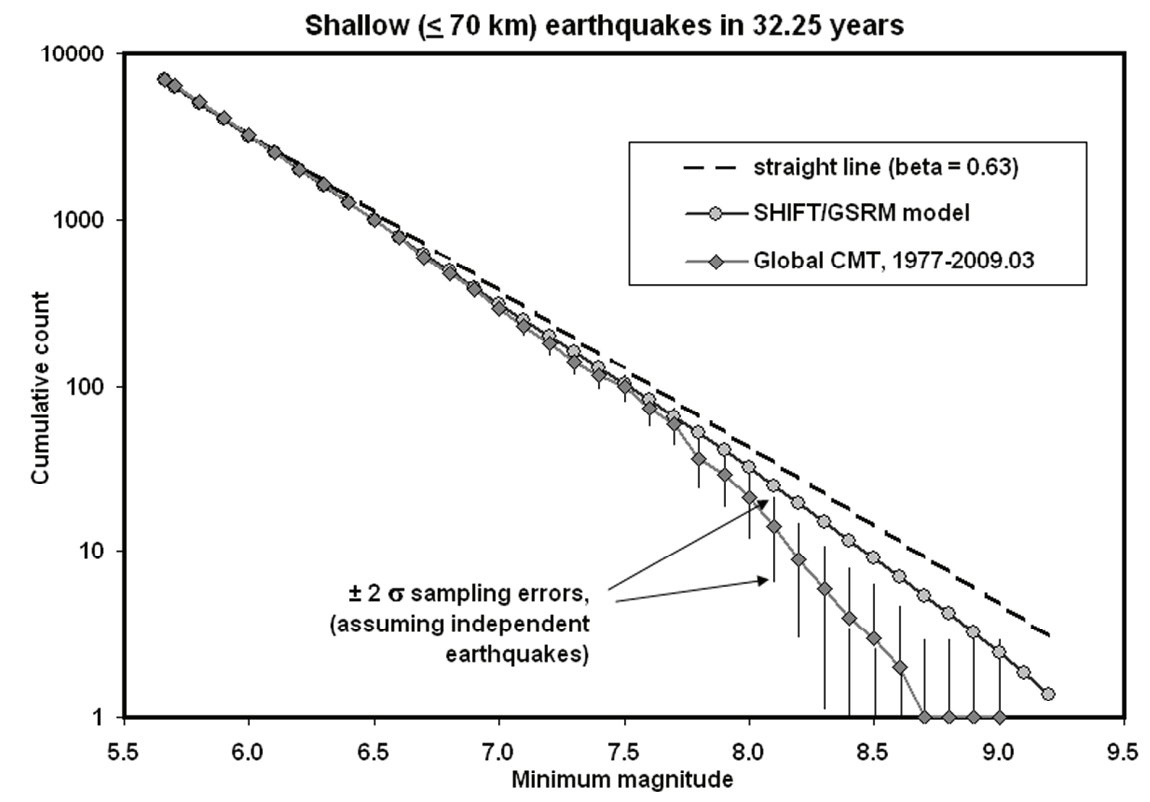

- Figure 5. Frequency/magnitude curve of the SHIFT/GSRM long-term shallow earthquake forecast, retrospectively compared to the CMT catalog of 1977-2009.03. No single tapered Gutenberg-Richter model is expected to fit this global composite of different tectonic regimes. Therefore a straight-line Gutenberg-Richter model with spectral slope $\beta=0.63$ is shown for comparison. Error bars on CMT earthquake counts are two-sigma sampling errors if and only if the distribution of earthquake counts follows the binomial distribution; actual sampling errors are probably larger due to earthquake clustering. 


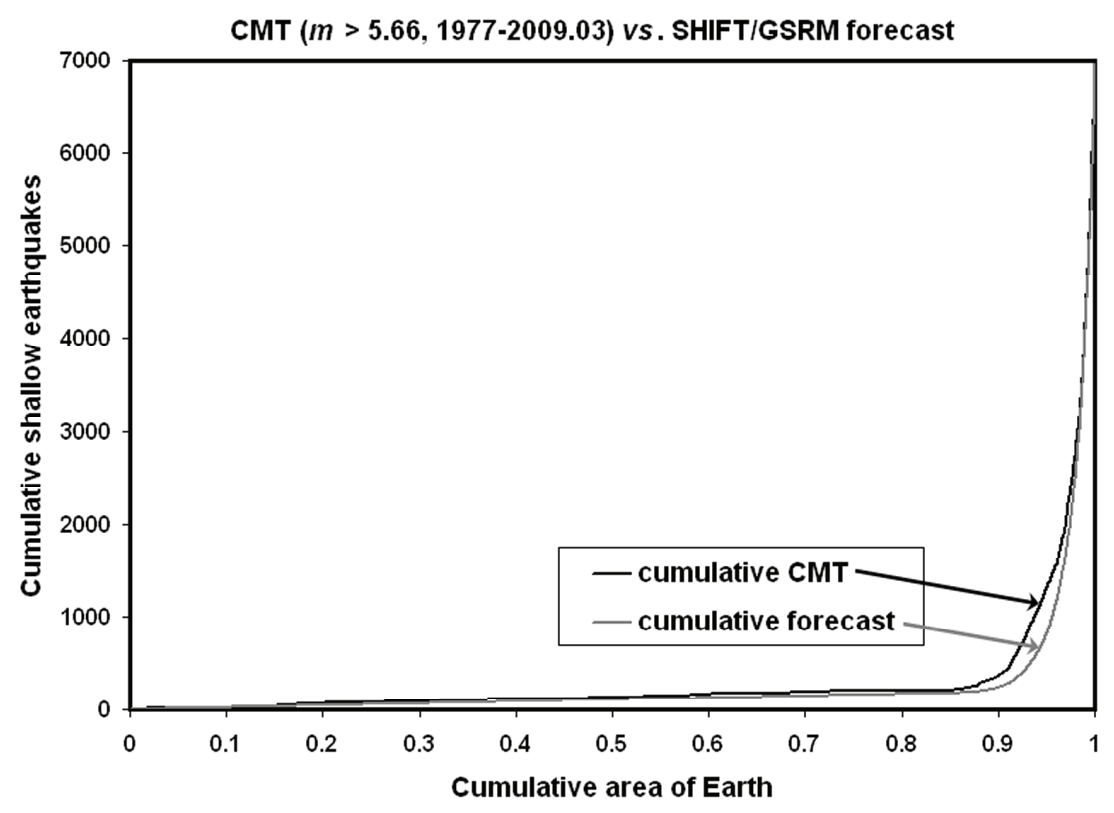

A Figure 6. Cumulative spatial distribution functions for both forecast and actual numbers of shallow earthquakes above $m_{T}=5.66$ in a 32.25 -year period. The abscissa is cumulative dimensionless area of Earth surface, relative to unity for the whole Earth. Grid cells with low forecast earthquake rates contribute to the left end of each curve, and grid cells with high forecast rates contribute to the right ends of each curve, as explained in text.

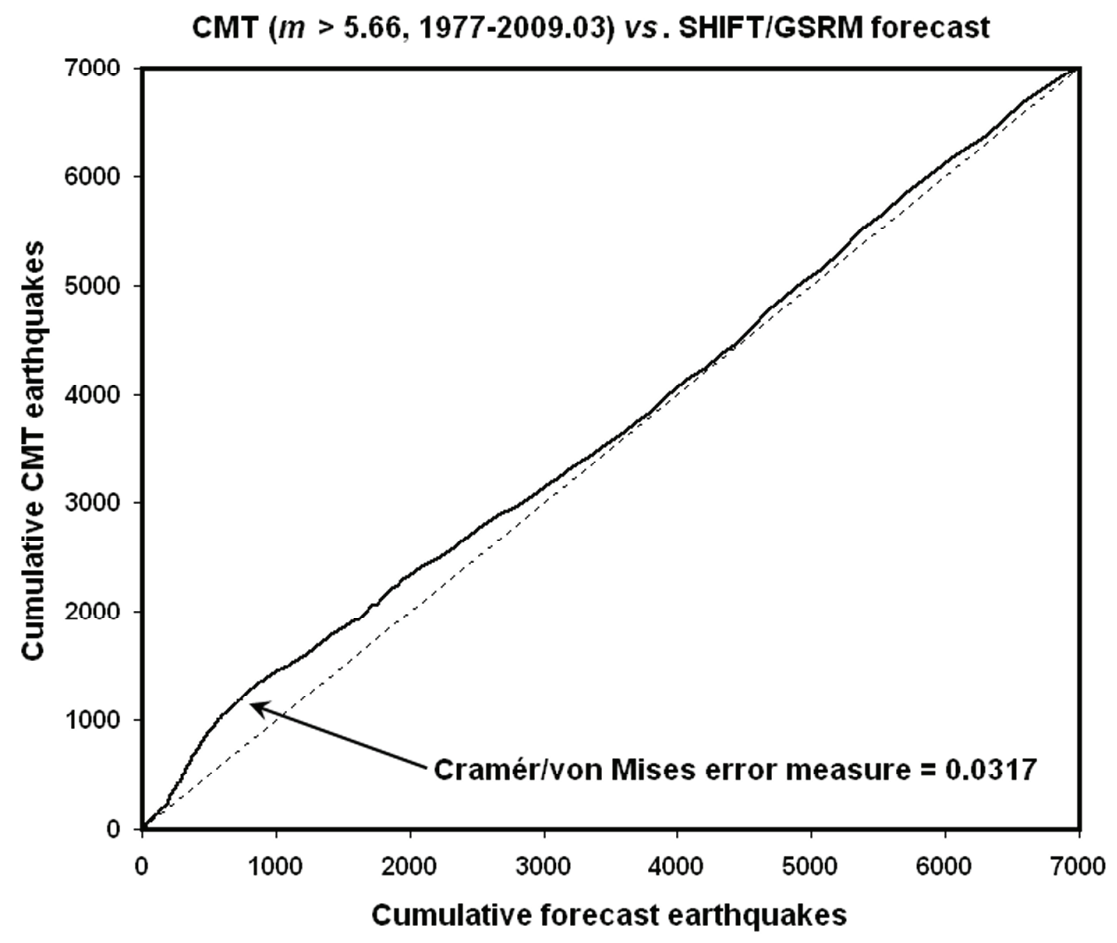

A Figure 7. Cumulative spatial distribution function for actual numbers of shallow earthquakes above $m_{T}=5.66$ in a 32.25 -year period (vertical axis; ordinate) is plotted against cumulative spatial distribution function of forecast numbers (horizontal axis; abscissa). Grid cells with low forecast earthquake rates contribute to the lower left end of the curve, and grid cells with high forecast rates contribute to the upper right ends of the curve, as explained in text. An ideal forecast would yield a straight line with slope of unity, as shown by the dotted line (except for small variations caused by finite-catalog sampling errors). See text for discussion of the discrepancy in the lower-left portion of the curve. The Cramér/von Mises error measure is the root-mean-square discrepancy between the curve and the diagonal when both axes are nondimensionalized to range $[0,1]$. 
verted, by convolution with chosen attenuation laws, to a set of maps of seismic hazard useful for long-term planning, building codes, and insurance. Naturally, it would then be necessary to consider and add the modest contributions to hazard from intermediate and deep earthquakes. $\mathbf{z}$

\section{ACKNOWLEDGMENTS}

This work was supported by the National Science Foundation under grant EAR-0711515 to the University of California.

\section{REFERENCES}

Anderson, J. G. (1979). Estimating the seismicity from geological structure for seismic-risk studies. Bulletin of the Seismological Society of America 69, 135-158.

Bird,P.(2003).Anupdated digitalmodelof plateboundaries, Geochemistry Geophysics Geosystems 4(3), 1027, doi:10.1029/2001GC000252.

Bird, P., Y. Y. Kagan, and D. D. Jackson (2002). Plate tectonics and earthquake potential of spreading ridges and oceanic transform faults, in: S. Stein and J. T. Freymueller (editors), Plate Boundary Zones, Geodynamics Series 30, 203-218, Am. Geophys. U., Washington, DC.

Bird, P., and Y. Y. Kagan (2004). Plate-tectonic analysis of shallow seismicity: Apparent boundary width, beta, corner magnitude, coupled lithosphere thickness, and coupling in seven tectonic settings. Bulletin of the Seismological Society of America 94 (6), 2,380-2,399, plus electronic supplement.

Bird, P., and Z. Liu (2007) Seismic hazard inferred from tectonics: California. Seismological Research Letters 78, 37-48.

Bird, P., Y. Y. Kagan, D. D. Jackson, F. P. Schoenberg, and M. J. Werner (2009). Linear and nonlinear relations between relative plate velocity and seismicity. Bulletin of the Seismological Society of America 99 (6), 3,097-3,113.

Ekström, G., A. M. Dziewonski, N. N. Maternovskaya, and M. Nettles (2005). Global seismicity of 2003: Centroid-moment-tensor solutions for 1,087 earthquakes. Physics of the Earth and Planetary Interiors 148 (2-4), 327-351.

Giardini, D., G. Grunthal, K. Shedlock, and P. Zhang (1999). Global Seismic Hazard Map, 1:35,000,000. Global Seismic Hazard Assessment Program, UN/International Decade of Natural Disaster Reduction, International Lithosphere Program.

Hanks, T. C., and H. Kanamori (1979). A moment magnitude scale. Journal of Geophysical Research 84, 2,348-2,350.

Holt, W. E., C. Kreemer, A. J. Haines, L. Estey, C. Meertens, G. Blewitt, and D. Lavallée (2005). Project helps constrain continental dynamics and seismic hazards. Eos, Transactions, American Geophysical Union 86 (41), 383, 387.

Jackson, D. D., and Y. Y. Kagan (1999). Testable earthquake forecasts for 1999. Seismological Research Letters 70, 393-403.

Jordan, T. H., M. Gerstenberger, M. Liukis, P. J. Maechling, D. Schorlemmer, S. Wiemer, and J. D. Zechar (2007). Collaboratory for the Study of Earthquake Predictability, Earth-prints, http:// hdl.handle.net/2122/2332.
Kagan, Y. Y. (forthcoming). Statistical distributions of earthquake numbers: Consequence of branching process, Geophys. J. Int., in press. (ms. available at http://moho.ess.ucla.edu/ kagan/nbd_subm.pdf)

Kagan, Y. Y. (2003). Accuracy of modern global earthquake catalogs. Physics of the Earth and Planetary Interiors 135 (2-3), 173-209; doi:10.1016/S0031-9201(02)00214-5.

Kagan, Y. Y., and D. D. Jackson (2000). Probabilistic forecasting of earthquakes. Geophysical Journal International 143, 438-453.

Kreemer, C., J. Haines, W. E. Holt, G. Blewitt, and D. Lavallée (2000). On the determination of a global strain rate model. Earth Planets Space 52 (10), 765-770.

Kreemer, C., W. E. Holt, and A. J. Haines (2002). The global moment rate distribution within plate boundary zones. In Plate Boundary Zones, ed. S. Stein and J. T. Freymueller, 173-190. Geodynamics Series 30. Washington, DC: American Geophysical Union.

Kreemer, C., W. E. Holt, and A. J. Haines (2003). An integrated global model of present-day plate motions and plate boundary deformation. Geophysical Journal International 154, 8-34.

Pacheco, J. F., and L. R. Sykes (1992). Seismic moment catalog of large, shallow earthquakes, 1900-1989. Bulletin of the Seismological Society of America 82, 1,306-1,349.

Pancha, A., J. G. Anderson, and C. Kreemer (2006). Comparison of seismic and geodetic scalar moment rates across the Basin and Range Province. Bulletin of the Seismological Society of America 96, 11-32.

Savage, J. C., and R. W. Simpson (1997). Surface strain accumulation and the seismic moment tensor. Bulletin of the Seismological Society of America 87, 1,345-1,353.

Ward, S. N. (1994). A multidisciplinary approach to seismic hazard in Southern California. Bulletin of the Seismological Society of America 84, $1,293-1,309$.

Ward, S. N. (1998a). On the consistency of earthquake moment rates, geological fault data, and space geodetic strain: The United States. Geophysical Journal International 134, 172-186.

Ward, S. N. (1998b). On the consistency of earthquake moment rates, geological fault data, and space geodetic strain: Europe. Geophysical Journal International 135, 1,011-1,018.

Department of Earth and Space Sciences
University of California
Los Angeles, California 90045 U.S.A.
pbird@ess.ucla.edu

(P. B.)

Nevada Bureau of Mines and Geology

University of Nevada

Reno, Nevada 89557-0178 U.S.A.

kreemer@unr.edu

(C.K.)

Department of Geosciences

State University of New York

Stony Brook, New York 11794-2100 U.S.A.

william.holt@sunysb.edu

(W.E.H.) 